\title{
Airway neutrophilia in stable and bronchiolitis obliterans syndrome patients following lung transplantation
}

Ling Zheng, E Haydn Walters, Chris Ward, Ning Wang, Bernadette Orsida, Helen Whitford, Trevor J Williams, Tom Kotsimbos, Gregory I Snell

\begin{abstract}
Background-The bronchiolitis obliterans syndrome (BOS) remains the major constraint on the long term success of lung transplantation. Neutrophils have been associated with fibrosing lung conditions and have been noted to be increased in the bronchoalveolar lavage (BAL) fluid of patients with BOS.
\end{abstract}

Methods-This study was undertaken to examine neutrophil accumulation in the BAL fluid, airway wall and lung parenchyma, as well as levels of interleukin (IL) -8 in the BAL fluid, in normal controls and lung transplant recipients with and without BOS. Bronchoscopic examination included endobronchial biopsy (EBB), BAL fluid, and transbronchial biopsy (TBB) sampling. Tissue neutrophils were identified by neutrophil elastase staining on $3 \mu \mathrm{m}$ paraffin biopsy sections and quantified by computerised image analyser. IL-8 levels were measured in unconcentrated BAL fluid by ELISA.

Results-Compared with controls, airway wall neutrophilia was increased in both stable lung transplant recipients and those with BOS $(p<0.05)$. BAL neutrophils and IL-8 levels were also increased in both groups of transplant recipients compared with controls $(p<0.01)$, the levels being significantly higher in the BOS group $(p<0.01)$. Neutrophil numbers in the lung parenchyma were not significantly different between the two groups of lung transplant recipients.

Department of Respiratory Medicine, Alfred Hospital and Monash Medical

School, Prahran 3181, Melbourne, Australia L Zheng

E H Walters

C Ward

N Wang

B Orsida

$\mathrm{H}$ Whitford

T J Williams

T Kotsimbos

G I Snell

Correspondence to:

Dr G Snell

Received 22 March 1999

Returned to authors

2 June 1999

Revised manuscript received

29 July 1999

Accepted for publication

4 October 1999
$50 \%$ of recipients within the first 18 months after lung transplantation. ${ }^{1}$ BOS is characterised clinically by progressive dyspnoea and a decline in the airflow parameters of pulmonary function testing and histologically by obliteration of the bronchiolar lumen either by polyps of connective tissue or by submucosal fibrosis and scarring. ${ }^{2-4}$ It is our current understanding that BOS is the clinical manifestation of histopathological chronic rejection of the lung allograft, and there is increasing evidence that chronic lung rejection is a pan-airway process, affecting large as well as small airways. ${ }^{5-7}$

Although the mechanisms by which chronic lung rejection lead to BOS still remain unclear, alloreactive $\mathrm{T}$ cells, pulmonary macrophage and adaptive immune mechanisms have always been the centrepiece of models of the pathogenesis of BOS and the main target of standard immunosuppression regimes. ${ }^{49}$ By contrast, there has been relatively little consideration given to the role that excessive innate, nonspecific, allo-independent mechanisms of injury may play in the development of chronic rejection in the lung allograft.

There is increasing evidence that persistent neutrophilic inflammation is associated with a number of fibrosing inflammatory pulmonary conditions including cryptogenic fibrosing alveolitis and asbestosis, and in a diverse range of lung models of induced pulmonary fibrosis. ${ }^{10-13}$ Neutrophilic inflammation has also been noted in the airways of patients with severe asthma requiring steroids. ${ }^{14}$ It has been shown that an increase in the proportion and total number of neutrophils in the bronchoalveolar lavage (BAL) fluid ${ }^{15}$ and transbronchial biopsy (TBB) specimens occurs in lung transplant recipients with BOS. ${ }^{17}$ DiGiovine et al have also shown that the increased neutrophilia in the BAL fluid of lung transplant recipients with BOS is associated with increased interleukin (IL)-8 levels in the BAL fluid and that IL- 8 accounts for a large portion of the neutrophil chemotactic activity in these subjects. ${ }^{15}$ Furthermore, their airway tissue immunostaining localises to peribronchial tissues. These results are in keeping with previous data that suggested that airway neutrophilia in inflammatory lung disease is strongly linked with IL-8. ${ }^{1018}$ The clinical relevance of these observations has recently been confirmed in a small retrospective series by Henke and co-workers who showed that persistent BAL neutrophilia predicted mortality after lung transplantation. ${ }^{20}$

We hypothesised that chronic neutrophilic inflammation is present in all three compartments (airway wall, lung parenchyma, and 
BAL fluid) of lung allografts that have developed BOS, consistent with the evidence that BOS is part of a pan-airway chronic rejection phenomenon. We further speculated that this neutrophilic inflammation is predominant in the small airways, which are the physiological site of airflow limitation in BOS, and that this accumulation of neutrophils is not primarily linked to overt infection of the lung allograft. Our previous work suggested that BAL fluid and endobronchial biopsy (EBB) specimens may give complementary information about airway inflammation beyond that demonstrable with TBB alone and, indeed, that BAL may have some advantages in sampling small airways. ${ }^{15}$

\section{Methods}

STUDY POPULATION AND INCLUSION CRITERIA

Twenty nine lung transplant recipients were studied (table 1); 19 were clinically stable 58-1585 days after transplantation, sustaining their best postoperative lung function (mean (95\% confidence interval) percentage best forced expiratory volume in one second $\left(\mathrm{FEV}_{1}\right)$ after transplantation $98.9(1.9) \%$ ) and without evidence of acute allograft rejection or significant airway infection in the one month prior to the study. Ten were diagnosed as having BOS and were studied 252-1518 days after transplantation. The diagnosis of BOS was based on the criterion of a decline in $\mathrm{FEV}_{1}$ of at least $20 \%$ from their best postoperative lung function (mean percentage best $\mathrm{FEV}_{1}$ after transplantation $52.2(9.5) \%$ in the absence of any

Table 1 Clinical details of lung transplant recipients (LTR) with and without bronchiolitis obliterans syndrome (BOS)

\begin{tabular}{|c|c|c|c|c|c|c|c|}
\hline No & Sex & $\begin{array}{l}\text { Age } \\
\text { (years) }\end{array}$ & $\begin{array}{l}\text { Original } \\
\text { disease* }\end{array}$ & $L T x$ & $\begin{array}{l}\text { Days after } \\
\text { LTx }\end{array}$ & $\begin{array}{l}\text { TBB airway } \\
\text { grading\# }\end{array}$ & $\begin{array}{l}\% \text { best FEV } \\
\text { (mean }(C I))\end{array}$ \\
\hline \multicolumn{8}{|c|}{ LTR without BOS } \\
\hline 1 & $\mathrm{~F}$ & 47 & B & BSLTx & 107 & $\mathrm{Bx}$ & 100 \\
\hline 2 & M & 40 & B & BSLTx & 189 & B0 & 100 \\
\hline 3 & $\mathrm{~F}$ & 31 & $\mathrm{CF}$ & BSLTx & 58 & B2 & 100 \\
\hline 4 & M & 38 & $\mathrm{E}$ & HLTx & 1185 & B0 & 100 \\
\hline 5 & $M$ & 22 & $\mathrm{CF}$ & BSLTx & 186 & $\mathrm{Bx}$ & 100 \\
\hline 6 & $M$ & 42 & $\mathrm{E}$ & HLTx & 1090 & B0 & 99 \\
\hline 7 & $M$ & 38 & $\mathrm{CF}$ & BSLTx & 762 & B0 & 100 \\
\hline 8 & $\mathrm{~F}$ & 35 & $\mathrm{E}$ & HLTx & 387 & B0 & 96 \\
\hline 9 & $\mathrm{~F}$ & 43 & PPH & HLTx & 369 & B2 & 100 \\
\hline 10 & M & 49 & EM, VHD & HLTx & 183 & Bo & 100 \\
\hline 11 & $\mathrm{~F}$ & 35 & PPH & BSLTx & 287 & $\mathrm{Bx}$ & 96 \\
\hline 12 & $\mathrm{~F}$ & 37 & $\mathrm{E}$ & HLTx & 464 & B0 & 100 \\
\hline 13 & $\mathrm{~F}$ & 23 & $\mathrm{CF}$ & BSLTx & 102 & B0 & 100 \\
\hline 14 & $\mathrm{~F}$ & 23 & $\mathrm{E}$ & HLTx & 1144 & B0 & 100 \\
\hline 15 & $M$ & 46 & Sar & BSLTx & 1276 & B2 & 100 \\
\hline 16 & $\mathrm{~F}$ & 42 & $\mathrm{E}$ & HLTx & 1585 & $\mathrm{Bx}$ & 100 \\
\hline 17 & $\mathrm{~F}$ & 32 & $\mathrm{CF}$ & BSLTx & 67 & B0 & 100 \\
\hline 18 & M & 33 & $\mathrm{~B}$ & BSLTx & 560 & B2 & 89 \\
\hline 19 & M & 40 & $\mathrm{CF}$ & BSLTx & 547 & Bo & 100 \\
\hline \multicolumn{8}{|c|}{ LTR with BOS } \\
\hline 1 & $M$ & 24 & $\mathrm{CF}$ & HLTx & 1176 & B0 & 51 \\
\hline 2 & $M$ & 23 & $\mathrm{CF}$ & HLTx & 1124 & B0 & 64 \\
\hline 3 & M & 26 & $\mathrm{CF}$ & BSLTx & 252 & $\mathrm{Bx}$ & 72 \\
\hline 4 & $M$ & 26 & $\mathrm{CF}$ & BSLTx & 531 & B2 & 55 \\
\hline 5 & $\mathrm{~F}$ & 31 & PPH & BSLTx & 396 & B1 & 21 \\
\hline 6 & $\mathrm{~F}$ & 54 & E & SLTx & 1186 & B3 & 49 \\
\hline 7 & $\mathrm{~F}$ & 47 & EM & SLTx & 1518 & B2 & 28 \\
\hline 8 & $\mathrm{~F}$ & 26 & $\mathrm{E}$ & BLTx & 989 & $\mathrm{Bx}$ & 58 \\
\hline 9 & $\mathrm{~F}$ & 55 & $\mathrm{E}$ & SLTx & 582 & $\mathrm{~B} 1$ & 46 \\
\hline 10 & M & 28 & CF & BSLTx & 990 & B2 & $\begin{array}{l}50 \\
(494(9.5))\end{array}$ \\
\hline
\end{tabular}

${ }^{\star}$ Original disease: $\mathrm{B}=$ bronchiectasis $; \mathrm{CF}=$ cystic fibrosis; $\mathrm{E}=$ Eisenmenger's syndrome; $\mathrm{PPH}=$ primary pulmonary hypertension; $\mathrm{EM}=$ emphysema; $\mathrm{VHD}=$ valvular heart disease; Sar = sarcoidosis.

LTx $=$ lung transplant procedure; BSLTx $=$ bilateral sequential lung transplantation; HLTx $=$ heart-lung transplantation; SLTx = single lung transplantation.

\#Airway rejection defined as per ISHLT grading system. ${ }^{30}$ other detectable complication that could cause a deterioration in lung function).$^{20}$

Recruitment required the absence of any significant airway infection-that is, afebrile, no new chest crepitations or consolidation, unchanged leucocyte count, and unchanged chest radiograph. In addition, there had to be no evidence of acute bronchitis or mucopus on bronchoscopic assessment of the airways. Any micro-organisms that were isolated from the BAL fluid had to be considered microbiologically as colonisers and clinically as non-disease producing and hence not requiring treatment. More specifically, gram stains for bacteria were negative in all cases and cytomegalovirus cellular inclusions were not detected in any of the BAL fluid or biopsy samples.

All patients were receiving a standard long term maintenance regimen of immunosuppressive therapy comprising cyclosporin (to achieve a blood level of 200-350 $\mu \mathrm{g} / \mathrm{l}$ by EMIT assay; Syva, California, USA), azathioprine (1-2 mg/kg/day), and prednisolone (0.15$0.25 \mathrm{mg} / \mathrm{kg} /$ day). Surgical techniques and preservation methods were standard within operative groups and were as described elsewhere. ${ }^{21}$

Twenty normal non-smoking volunteer subjects (15 men of mean (95\% CI) age 29 (6) years and five women of mean age 26 (3.5) years) were recruited as controls. They were asymptomatic with normal lung function.

Approval for the study was given by the Alfred Hospital Ethics Committee and each subject gave written informed consent. Haemodynamic status and oxygen saturation levels were closely monitored throughout the bronchoscopic procedure.

BRONCHOALVEOLAR LAVAGE (BAL),

TRANSBRONCHIAL BIOPSY (TBB) AND

ENDOBRONCHIAL BIOPSY (EBB)

BAL fluid and TBB and EBB specimens were obtained at routine bronchoscopic surveillance performed under intravenous sedation with midazolam (Roche, Paris, France). In our institution this procedure is carried out at 14 days, $1,2,3,6,9,12,18$ and 24 months after transplantation, and then annually.

For BAL, a total of $180 \mathrm{ml}$ warmed $\left(37^{\circ} \mathrm{C}\right)$ phosphate buffered saline solution was instilled in the right middle lobe or lingula in three $60 \mathrm{ml}$ aliquots with immediate aspiration at a negative pressure of approximately $-80 \mathrm{~mm} \mathrm{Hg}$ after each instillation. On average, six EBB specimens were taken as extra tissue samples for research purposes from lower lobe subcarinae using alligator forceps (Olympus FB 15C, Tokyo, Japan). TBB specimens were taken as part of the normal follow up protocol for lung transplant recipients, usually from the lower lobe. The normal controls underwent the same procedure of BAL and $\mathrm{EBB}$ on a single occasion, but not TBB for ethical reasons.

Ten $\mathrm{ml}$ pooled BAL fluid was retained for microbiological testing, the rest being immediately transported to the laboratory at $4^{\circ} \mathrm{C}$ for processing and analysis. The BAL fluid total cell count was determined using a Neubauer counting chamber with unfiltered fluid. Cytospin preparations were made using a Shandon 
Cytospin III centrifuge with $200 \mu$ unfiltered BAL fluid spun at $850 \mathrm{rpm}$ for 10 minutes and stained with "Quik Diff”. BAL fluid cell differential counts were performed on duplicate cytospin preparations by counting 1000 cells. The supernatants were obtained by filtering the pooled BAL fluid through a $200 \mu \mathrm{m}$ nylon mesh, centrifuging at $1500 \mathrm{rpm}$ for $10 \mathrm{~min}-$ utes, and then aliquoting into $1.5 \mathrm{ml}$ tubes to be stored at $-80^{\circ}$ until used. EBB and TBB specimens were snap frozen in a liquid nitrogen chilled isopentane slurry, embedded with ornithine carbamyl transferase (OCT), and stored at $-80^{\circ} \mathrm{C}$.

\section{IMMUNOHISTOCHEMISTRY}

Immunohistological staining for neutrophil elastase was performed using a monoclonal mouse anti-human neutrophil elastase antibody (Dako, Denmark) and amplified using an avidin-biotin peroxidase complex (ABC) method. Isotype control immunoglobulins ( $\operatorname{IgG}_{1}$, Dako, Denmark) were used as negative controls. EBB and TBB specimens were post-processed into $10 \%$ formalin for two hours and embedded in paraffin using an automated tissue processor (Histokinette, Shandon). Serial consecutive $3 \mu \mathrm{m}$ sections were cut from the post-processed tissue and deparaffinised in xylene, rehydrated through graded ethanol solutions, and washed with TBS. The quality of the sections thus obtained was assessed using standard haematoxylin and eosin staining. The sections were then incubated for 20 minutes with $0.015 \%$ normal horse serum in TBS to prevent non-specific binding of immunoglobulins to tissue, covered with anti-neutrophil elastase antibody (diluted $1: 600$ in TBS) at $4^{\circ} \mathrm{C}$ in a moist chamber overnight, and then washed again with TBS. The sections were incubated with biotinylated horse anti-mouse antibody (Vector Laboratories, California, USA) for 30 minutes, treated with $0.12 \%$ hydrogen peroxide for 10 minutes to block endogenous peroxidase activity, and then incubated for 30 minutes with a preformed avidin and biotinylated horseradish-peroxidase macromolecular complex (VectaStain Elite ABC kit, Vector Laboratories Burlingame, California, USA) using diaminobenzidine (Dako, Denmark) as substrate. EBB sections were then counterstained with haematoxylin and mounted in synthetic mounting medium. TBB sections, after counterstaining with haematoxylin, were counterstained again with $2 \%$ light green for the connective tissue which allowed a determination of the total area of alveolar septa. The slides were then mounted in synthetic mounting medium.

To minimise subjective variation in interpretation of the slides, staining was all done by one person. Slides were coded and then assessed by a single blinded observer. The total neutrophil elastase positive cells in the lamina propria of the airway wall and lung parenchyma in the EBB and TBB sections, respectively, were counted by using a computerised image analyser (Video Pro 32, Leading Edge, Sydney, Australia) at a final magnification of $\times 400$. Positive elastase staining was scored if it was exclusively intracellular within neutrophils. Results were expressed as positive cells $/ \mathrm{mm}^{2}$ lamina propria or positive cells $/ \mathrm{mm}^{2}$ alveolar septa.

IL-8 ASSAY

BAL fluid concentrations of IL-8 were measured in unconcentrated fluid with an enzyme-linked immunosorbent assay (ELISA) using a commercially available kit with precoated microtitre plates (Amersham, UK). The detection range was $10-1000 \mathrm{pg} / \mathrm{ml} .50 \mu \mathrm{l}$ of the standard and unconcentrated BAL supernatants were incubated in duplicate in the microtitre plates. After washing away any unbound sample proteins, a biotinylated antibody to IL-8 was added to the wells, followed by another wash and incubation with streptavidin conjugated to horseradish peroxidase. After adding the substrate, colour developed in proportion to the amount of IL- 8 bound in the initial step. The absorbency was measured in an ELISA reader (Model 450, Bio-Rad, USA) at $450 \mathrm{~nm}$. The amount of IL-8 present in the samples was calculated by reference to the wells containing dilutions of the standard for IL-8.

\section{LUNG FUNCTION}

Lung function tests used for analysis were performed in all the patients immediately before bronchoscopy. A computerised rolling seal spirometer (Sensor Medics 922, California, USA) was used to measure flow-volume loops. As part of the routine follow up following transplantation, regular spirometric tests were performed with the best forced expiratory volume in one second $\left(\mathrm{FEV}_{1}\right)$ after surgery being used as the benchmark standard against which to assess later development of BOS.

\section{STATISTICAL ANALYSIS}

BAL data and biopsy data are expressed as means with the $95 \%$ confidence interval (CI) shown in parentheses. Where a range is stated this refers to the absolute range of the raw data. All comparisons between groups were made using the non-parametric Mann-Whitney rank order test. Correlations were evaluated by Spearman's rank method. $p$ values of $\leqslant 0.05$ were considered as statistically significant.

\section{Results}

NEUTROPHILS IN THE AIRWAY WALL

Adequate EBB specimens for neutrophil elastase staining were obtained from 12 of 19 lung transplant recipients without BOS, seven of 10 with BOS, and 12 normal controls. Neutrophil distribution was mainly beneath the epithelium, although some were seen in the epithelium itself. Interestingly, in some of the specimens from lung transplant recipients neutrophil adhesion was also observed along the endothelium in the lumen of the airway vasculature.

The mean airway wall neutrophil count in the normal controls was 220 (104) neutrophils/ $\mathrm{mm}^{2}$ lamina propria (range 47-691). Compared with the normal controls the neutrophil count in the EBB specimens was increased significantly in lung transplant recipients without BOS (450 (128) neutrophils $/ \mathrm{mm}^{2}$ lamina pro- 


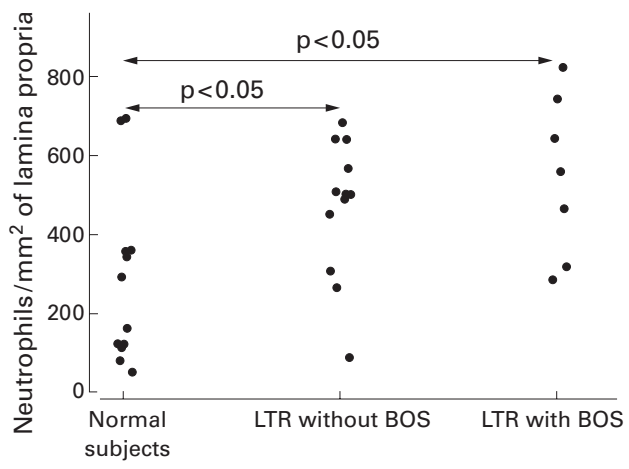

Figure 1 Neutrophil elastase positive cells in the endobronchial biopsy specimens from normal controls, lung transplant recipients (LTR) without BOS, and LTR with BOS.

pria, range $94-701, \mathrm{p}<0.05)$ but was even more prominently increased in those with BOS (557 (149) neutrophils $/ \mathrm{mm}^{2}$ lamina propria, range 299-832, p<0.05; fig 1).

NEUTROPHILS IN THE LUNG PARENCHYMA Although all patients had TBB tissue sufficient to grade for acute rejection for routine clinical histopathological examination (table 1), adequate additional TBB specimens were available for this study in 11 of the 19 lung transplant recipients without BOS and in five of the 10 recipients with BOS. Further, given that not all TBB specimens had adequate bronchiolar tissue for specific assessment of neutrophils, further analysis relates to the parenchymal structures only. The distribution of neutrophil elastase positive cells in the TBB specimens was mainly present in the alveolar septa. This contrasted with the distribution of CD68 positive cells which were easily seen in the alveolar space (data not shown). The number of neutrophils $/ \mathrm{mm}^{2}$ of alveolar septa in transplant recipients with BOS (1033 (480), range 903-2376) was similar to that in those without BOS (1363 (407), range 368-2470).

NEUTROPHILS IN BAL FLUID

BAL fluid data and cell profiles are summarised in table 2. Only seven patients with BOS underwent adequate BAL due to oxygen desaturation in two subjects which led to termination of the bronchoscopic procedure on clinical grounds. All 19 lung transplant recipients without BOS and 20 normal controls underwent an adequate BAL procedure; the returns were significantly different between the groups. The mean return for the lung transplant recipients with BOS was 63 (22) $\mathrm{ml}$ (range 26-95) compared with 117 (5) $\mathrm{ml}$ in normal controls $(\mathrm{p}<0.001)$. In those without BOS the mean return was 86 (11) $\mathrm{ml}$ (range $40-112),(p<0.001$ versus normal controls and $\mathrm{p}<0.05$ versus lung transplant recipients with BOS).

The most striking feature of the BAL fluid data was a significant increase in neutrophils in the BOS group, both in the percentage $(\mathrm{p}<0.001$ versus both normal controls and lung transplant recipients without BOS, table 2 ) and in the absolute counts $\left(55(69) \times 10^{4} / \mathrm{ml}\right.$, range 12-1643) compared with both normal subjects $\left(14\right.$ (2) $\times 10^{4} / \mathrm{ml}$, range $1-13$; $\mathrm{p}<0.001)$ and transplant recipients without BOS $\left(16(5) \times 10^{4} / \mathrm{ml}\right.$, range $\left.1-74, \mathrm{p}<0.001\right)$. The neutrophil count in the transplant recipients with BOS, though raised to a more modest extent, was also significantly different from the normal subjects $(p<0.001)$. Reciprocally, the proportion of macrophages was lower in subjects with BOS than in normal controls $(p<0.001)$ and in transplant recipients without BOS $(\mathrm{p}<0.01$, table 2$)$.

BAL IL-8 LEVELS

The BAL levels of IL- 8 in the normal controls was $27.5(7.5) \mathrm{pg} / \mathrm{ml}$ (range 8.7-84.6). Compared with normal subjects the IL-8 levels were markedly increased in BAL fluid from lung transplant recipients with BOS (558 (423) pg/ $\mathrm{ml}$, range 36-1076, $\mathrm{p}<0.001$ ) and, to a lesser extent, in BAL fluid from transplant recipients without BOS (84.2 (53) pg/ml, range 17.4-449, $\mathrm{p}<0.01$, fig 2). The difference in IL-8 levels between the lung transplant groups was significant $(p<0.01)$. The IL-8 levels in BAL fluid from those with BOS was strongly correlated with the percentage of neutrophils in the BAL fluid ( $r=0.82, \mathrm{p}<0.05$, fig 3 ). In contrast, IL-8 levels in lung transplant recipients without BOS were not correlated with the percentage of neutrophils in the BAL fluid $(r=0.29, \mathrm{p}=0.22)$.

AIRWAY WALL, LUNG PARENCHYMA, AND BAL CORRELATIONS

Seventeen of the 19 lung transplant recipients with adequate EBB specimens had paired BAL fluid data that could be analysed further (12/12 without BOS, 5/7 with BOS). In transplant recipients without BOS there was no correlation between airway wall neutrophilia, lung parenchyma, and BAL neutrophilia. In those with BOS there was a correlation between airway wall and BAL neutrophilia $(r=0.58)$ that was not statistically significant with the small number of subjects. The results did not correlate with the BOS staging nor with the time between the study date and deterioration from BOS stage 0 .

Table 2 BAL fluid recovery and cellular composition in lung transplant recipients (LTR) with and without bronchiolitis obliterans syndrome (BOS) and normal controls

\begin{tabular}{llllllll}
\hline & $\begin{array}{l}\text { BAL return } \\
(\mathrm{ml})\end{array}$ & $\begin{array}{l}\text { TCC } \\
\left(\times 10^{4} / \mathrm{ml}\right)\end{array}$ & AM (\%) & Lym (\%) & Neu (\%) & Eos (\%) & Ep (\%) \\
\hline LTR with BOS $(\mathrm{n}=7)$ & $63(22)^{\star \star \star} \dagger$ & $55(69)$ & $36(19)^{\star \star \star}+\dagger$ & $17(12)$ & $42(27)^{\star \star \star}+\dagger$ & $0.8(0.6)$ & $4.0(3.1)$ \\
LTR without BOS $(\mathrm{n}=19)$ & $86(11)^{\star \star \star}$ & $16(5)$ & $73(8)$ & $19(9)$ & $4.7(2.2)^{\star \star}$ & $0.3(0.2)$ & $2.8(1.6)$ \\
Normal (n $=20)$ & $117(5)$ & $14(2)$ & $73(7)$ & $20(6)$ & $2.1(0.5)$ & $1.1(1.1)$ & $2.9(2.3)$ \\
\hline
\end{tabular}

Values are mean $(95 \% \mathrm{CI})$

TCC = BAL fluid total cell count; AM = alveolar macrophages; Lym = lymphocytes; Neu = neutrophils; Eos = eosinophils; Ep = epithelial cells.

${ }^{\star} \mathrm{p}<0.05$ vs normal subjects; ${ }^{\star \star} \mathrm{p}<0.01$ vs normal subjects; ${ }^{\star \star \star} \mathrm{p}<0.001$ vs normal subjects.

$\dagger \mathrm{p}<0.05$ vs LTR without BOS; $\dagger+\mathrm{p}<0.01$ vs LTR without BOS. 


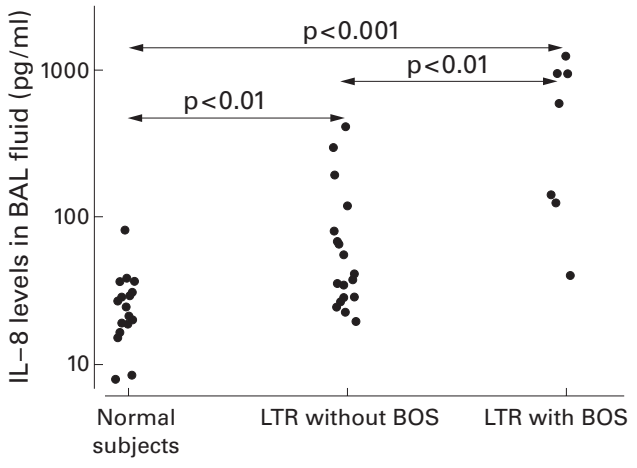

Figure 2 IL-8 levels ( $\mathrm{pg} / \mathrm{ml}$ ) in BAL fuid from normal controls, lung transplant recipients (LTR) without BOS, and LTR with BOS.

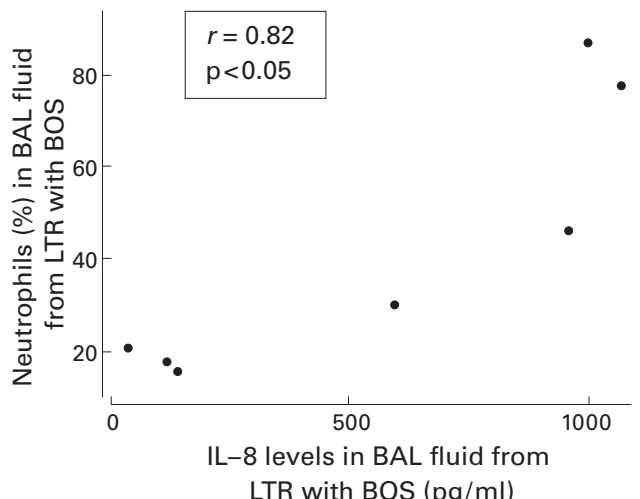

Figure 3 Correlation between percentage of neutrophils in $B A L$ fluid and IL-8 levels in lung transplant recipients (LTR) with BOS.

NEUTROPHILS AND THE ABILITY TO CULTURE ORGANISMS FROM BAL FLUID

As previously stated, lung transplant recipients were selected on the basis of there being no evidence of clinical infection. However, careful microbiological assessment isolated bacteria from BAL fluid on several occasions. Six of 19 recipients without BOS and five of 10 with BOS had positive bacterial cultures from BAL fluid and this difference was not statistically significant. The relationships between a positive BAL culture and BAL and biopsy findings are summarised in table 3 . From these data it is evident that, in lung transplant recipients without BOS, the percentage of neutrophils in BAL fluid and numbers of neutrophils in EBB specimens were not increased when organisms could be cultured in the BAL fluid. However, in lung transplant recipients with BOS, organ-

Table 3 Comparison of neutrophil (Neu) levels in lung transplant recipients (LTR) with and without bronchiolitis obliterans syndrome (BOS) based on the presence of organisms cultured from the BAL fluid

\begin{tabular}{|c|c|c|c|}
\hline & $B A L(\mathrm{Neu} \%)$ & $E B B\left(\mathrm{Neu} / \mathrm{mm}^{2}\right)$ & $T B B\left(\mathrm{Neu} / \mathrm{mm}^{2}\right)$ \\
\hline \multicolumn{4}{|c|}{ LTR with BAL bacteria $(\mathrm{n}=11)$} \\
\hline $\operatorname{BOS}(\mathrm{n}=5)$ & $\begin{array}{l}59(29)^{\star} \\
(\mathrm{n}=5)\end{array}$ & $\begin{array}{l}697(114)^{\star \star} \dagger \\
(\mathrm{n}=4)\end{array}$ & $\begin{array}{l}963(203) \\
(\mathrm{n}=3)\end{array}$ \\
\hline No BOS $(n=6)$ & $\begin{array}{l}3.4(1)^{\star} \\
(\mathrm{n}=6)\end{array}$ & $\begin{array}{l}437(100) \\
(\mathrm{n}=4)\end{array}$ & $\begin{array}{l}1102(621) \\
(\mathrm{n}=3)\end{array}$ \\
\hline \multicolumn{4}{|c|}{ LTR without BAL bacteria $(\mathrm{n}=18)$} \\
\hline $\operatorname{BOS}(n=5)$ & $\begin{array}{l}28(13)^{\star} \\
(\mathrm{n}=5)\end{array}$ & $\begin{array}{l}367(99) \dagger \\
(\mathrm{n}=3)\end{array}$ & $\begin{array}{l}1661(1401) \\
(n=2)\end{array}$ \\
\hline No BOS $(n=13)$ & $\begin{array}{l}5(3)^{\star} \\
(\mathrm{n}=13)\end{array}$ & $\begin{array}{l}452(229) \\
(\mathrm{n}=8)\end{array}$ & $\begin{array}{l}1341(524) \\
(\mathrm{n}=8)\end{array}$ \\
\hline
\end{tabular}

${ }^{\star} \mathrm{p}<0.02,{ }^{\star \star} \mathrm{p}<0.05$, lung transplant recipients with BOS vs without BOS.

$\mathrm{tp}<0.05$, lung transplant recipients with BOS with BAL bacteria vs without bacteria. isms in the BAL fluid were associated with a higher percentage of BAL neutrophils $(\mathrm{p}<0.05)$ and EBB neutrophilic infiltrate $(p<0.05)$. TBB neutrophil numbers were not influenced significantly by the ability to culture bacteria from BAL fluid.

\section{Discussion}

This study has, for the first time, shown quantitatively the relative neutrophil accumulation in the airway wall, BAL fluid, and lung parenchymal compartments of lung allografts. The study reveals a heavy neutrophil accumulation in the airway walls of lung transplant recipients, both with and without BOS, that was significantly higher than that seen in normal controls. BAL fluid neutrophil and IL-8 concentrations were also increased in both transplant groups, but more significantly in those with BOS. In the transplant recipients with BOS there was a significant correlation between airway wall neutrophilia and IL-8 concentrations. Where bacteria could be cultured from BAL fluid this did not seem to contribute to neutrophilia in the non-BOS population, although bacteria may have been influencing airway neutrophil counts in the BOS group.

BOS is generally viewed as an inflammatory airway condition and, although there were differences in BAL neutrophil numbers between transplant groups, it is striking that there was only a small difference in airway wall neutrophilia and no suggestion of excess neutrophils in the lung parenchyma of lung transplant recipients with BOS compared with those without BOS. The relative degree of lung parenchymal neutrophilia in transplant patients compared with a control population is not known as we have been unable to study appropriate tissue for ethical reasons. These findings are consistent with the work of DiGiovine et al who noted BAL neutrophilia as a marker of BOS in their population of lung transplant recipients yet found no correlation with the relatively few neutrophils seen in a small sample of open lung biopsy specimens from patients with BOS. ${ }^{15}$ However, their study had a low sensitivity for detection of tissue neutrophils as specific monoclonal antibody stains were not used and endobronchial biopsies were not studied. ${ }^{24}$ Our study using human neutrophil elastase immunostaining showed infiltration of considerable numbers of neutrophils in the lamina propria of the airway walls, as well as in the epithelium and subepithelial blood vessels - a distribution pattern easily overlooked by conventional haematoxylin eosin staining. ${ }^{24}$ In a further recent study Riise et al noted an association between BAL fluid neutrophils and immunostaining of TBB specimens for myeloperoxidase (a marker for neutrophils) in patients with BOS. ${ }^{17}$ These changes were confined to the bronchial walls, with alveolar and interstitial staining being no different from the non-BOS group. These studies emphasise the limitations of TBB as the traditional "gold standard" for the assessment of airway pathology.

It should be emphasised that BAL is likely to sample the distal airways at least as well as the 
alveolar spaces. Our data suggest that BAL fluid neutrophilia in lung allografts is mainly derived from the smaller airways and possibly lung parenchyma and, although there are some factors that cause neutrophil recruitment in both large and small airways (note the correlation between BAL fluid and EBB neutrophilia in lung transplant recipients with BOS), there are also factors that independently lead to neutrophil recruitment at the two sites (note the lack of a correlation between BAL fluid and EBB neutrophilia in transplant recipients without BOS). Airway wall neutrophilia suggests a chemokine gradient from epithelium to the vascular space. Our data implicate IL-8 as a driving force in this process and support the findings of other authors. ${ }^{15-17}$ DiGiovine et al localised IL-8 with immunostaining to the peribronchial regions of OB lesions and clearly showed that IL-8 accounted for a significant amount of the neutrophil chemotactic activity in their samples. ${ }^{15}$ This group also suggested that the development of BOS was predicted by a twofold increase in both neutrophils and IL-8 concentrations in the BAL fluid prior to a subsequent fall in lung function when compared with a long term stable non-BOS group. However, a major prospective longitudinal study is still necessary to clarify the importance of these relationships.

Before claiming a primary role for the neutrophil in the development of chronic lung rejection it is important that confounding by occult airway infection is considered, although this is not easily achieved. In our study, what would normally be regarded as significant airway infection was excluded by subject recruitment and specimen collection only occurring in the absence of overt infection, as assessed by clinical and microbiological criteria. Thus, all BAL samples were negative on gram staining and did not have cytomegalovirus inclusions, although organisms were still grown in some BAL fluid samples. The presence of positive bacterial culture results in the absence of a positive gram stain or clinical evidence of infection suggests that these bacteria were present in low numbers and were likely to be colonisers rather than causing active infection or disease. In this study organisms were not isolated more commonly from subjects with BOS than from those without the condition. Even so, the potential for subclinical bacterial infection as a co-factor in driving the airway inflammatory response remains to be considered in future studies.

In the BOS population the presence of organisms was associated with higher percentages of neutrophils in the BAL fluid and more neutrophils in EBB specimens, suggesting that occult infection may be important at this end of the spectrum. This has not been previously noted in other comparable studies which "excluded" patients with organisms. ${ }^{15}{ }^{17}$ However, the studies by DiGiovine et $a l^{15}$ (where lung transplant recipients were apparently excluded if any organisms were noted on BAL) and Riise $e t a l^{16}$ (where the BAL fluid bacterial load was used as a marker to exclude cases with infection) both reported a similar percentage of neutrophils in the BAL fluid as in the current study. A therapeutic role for prophylactic antibiotics may be worthy of future study, although neutrophilic infiltration in the lungs of smokers $^{25}$ and severe asthmatics ${ }^{14}$ is also associated with airway damage and airflow obstruction mimicking BOS without any current evidence of a role for infection, at least as presently defined.

When taken together, the increase in neutrophil numbers in the airway wall and BAL fluid from lung transplant recipients in this study leads to speculation that the infiltrating neutrophils participate in the development of airway fibrosis as part of the chronic rejection process following lung transplantation. Neutrophils contain potent inflammatory mediators-including proteases, acid hydrolases, low molecular weight cationic proteins, lipid secretory products, and reactive oxygen metabolites-which can potentially induce parenchyma cell injury and extracellular matrix degradation, thereby facilitating the development of pulmonary fibrosis. ${ }^{13}$ There is significant clinical and experimental evidence to support the development of a persistent neutrophil alveolitis preceding and playing an important part in the pathogenesis of pulmonary fibrosis. Since the early 1980s, with the introduction of BAL, it has been observed that patients with diffuse interstitial lung disease who had fibrosis had increased absolute and relative neutrophil counts in their lungs. ${ }^{13-20}$ Several studies have now reported a positive correlation between BAL neutrophilia and the clinical severity or activity of idiopathic pulmonary fibrosis (IPF). ${ }^{18} 1921$

However, the airway neutrophilia may be more important as a marker of excessive production of IL-8. IL-8 is a 72 amino acid polypeptide produced mainly by epithelial cells, interstitial fibroblasts, smooth muscle cells, and endothelial cells as well as alveolar macrophages and peripheral blood monocytes. ${ }^{16} 18$ Administration of IL-8 systemically to rabbits results in a significant granulocytosis, but when administered into the peritoneal space or intradermally it induces a marked local neutrophil influx..$^{19}$ IL-8 has been shown to be a neutrophil chemoattractant in conditions such as $\mathrm{IPF}^{1018}$ and, at nanomolar to millimolar concentrations, results in neutrophil activation. $^{18}$

However, IL- 8 has also been shown to be a potent angiogenic factor with an equivalent molar activity to more traditionally characterised angiogenic factors such as bFGF. IL-8 accounted for $42-80 \%$ of the overall angiogenic activity of freshly isolated human nonsmall cell lung cancer tumours in an ex vivo system. ${ }^{27}$ Conditioned media from fibroblasts isolated from open lung biopsy specimens from patients with IPF demonstrated constitutive angiogenic activity attributable, at least in part, to IL-8. ${ }^{28}$ Data from our laboratory have recently shown vascular changes in lung transplant recipients, both with and without BOS, with an exponential decrease in $\mathrm{FEV}_{1}$ as airway vessel size increased in those with BOS. ${ }^{29}$ Thus, it is likely that the multiple inflammatory and immunological activities of IL-8 would allow it 
to play a part in the pathophysiology associated with BOS at a number of levels. This is likely to involve both its role in neutrophil recruitment, but also directly through stimulating "airway remodelling" and particularly angiogenesis. $^{262729}$ In this context it is perhaps important that, in our study, IL-8 levels did not correlate with BAL fluid neutrophil counts in lung transplant recipients without BOS, indicating that IL-8 was perhaps uncoupled from a pure chemoattractant effect. BAL fluid levels of IL-8 can persist despite potent immunosuppressive regimes following lung transplantation ${ }^{15}{ }^{16}$ and a potential link between IL-8 and airway remodelling in lung transplant recipients clearly requires study in further longitudinal investigations.

In conclusion, we have shown that stable lung transplant recipients and those with BOS have increased airway wall neutrophilia, BAL fluid neutrophilia, and BAL fluid IL-8 levels. Airway neutrophilia cannot be accounted for by the presence of bacterial airway colonisation alone, although future studies must further define the role of subclinical infection as a co-factor for BOS development-for example, by stimulating IL-8 production in the airway. Endobronchial biopsy specimens continue to provide useful insights into airway immunopathology beyond what can be demonstrated with BAL and TBB alone. If longitudinal studies can confirm a link between airway neutrophils and/or increased IL-8 levels and the development of airway remodelling in BOS, then this creates novel potential targets for future therapeutic interventions.

Supported by a grant from The Alfred Foundation, Glaxo Wellcome Australia, and NHMRC Australia.

1 Sharples LD, Tamm RM, Mc Neill Ket al. Development of bronchiolitis obliterans syndrome in recipients of heartlung transplants: early risk factors. Transplantation 1996;61:560-6.

2 Maurer JR. Lung transplantation bronchiolitis obliterans. In: Epler GR, ed. Diseases of the bronchioles. New York: Raven Press, 1994: 275-89.

3 Hertz MI, Henke CA, Nakhleh ER, et al. Obliterative bronchiolitis after lung transplantation: a fibroproliferative chiolitis after lung transplantation: a fibroproliferative disorder associated with platelet-derived
Proc Natl Acad Sci USA 1992;89:10385-9.

4 Winter JBC, Clelland A, Gouw SH, et al. Distinct phenotypes of infiltrating cells during acute and chronic phenotypes of infiltrating cells during acute and chronic
lung rejection in human heart-lung transplants. Transplanlung rejection in hum

5 Zheng L, Ward C, Snell GI, et al. Scar collagen deposition in airways of lung transplant recipients. Am $\mathcal{F}$ Respir Crit Care Med 1997;55:2072-7.

6 Youssem SA, Duncan SR, Dauber JA, et al. Architectural remodelling of lung allografts in acute and chronic rejection. Arch Pathol Lab Med 1992;116:1175-80.
7 Magnan A, Mege JL, Escallier JC, et al. Balance between alveolar macrophage IL-6 and TGF- $\beta$ in lung-transplan recipients. Am f Respir Crit Care Med 1996;53:1431-6.

8 Pual LC, Benedisktsson H. Chronic transplant rejection: magnitude of the problem and pathogenetic mechanisms. Transplant Rev 1993;7:96-113.

9 Tullius SG, Tilney NL. Both alloantigen-dependent and -independent factors influence chronic allograft rejection. Transplantation 1995;59:313-8.

10 Lynch III JP, Standiford TJ, Rolfe MW, et al. Neutrophilic alveolitis in idiopathic pulmonary fibrosis: the role of interleukin-8. Am Rev Respir Dis 1992;145:1433-9.

11 Rola A, Pleszczynski M, Gouin S, et al. Asbestosis-induced lung inflammtion: role of local macrophage-derived chemotactic factors in accumulation of neutrophils in the lungs. Inflammation 1994;8:53-62.

12 Clark JK, Kuhn C. Bleomycin induced pulmonary fibrosis in hamsters: effect of neutrophil depletion on lung collagen in hamsters: effect of neutrophil depletion on lung

13 Brieland JK, Fantone JC. Neutrophils and pulmonary fibrosis. In: Phan SH, Thrall RS, eds. Pulmonary fibrosis. New Yis. In: Phan SH, Thrall RS, eds. Pulm

14 Wenzel SE, Szefler SJ, Leung DY, et al. Bronchoscopic evaluation of severe asthma: persistent inflammation associated with high dose glucocorticoids. Am F Respir Crit Care Med 1997;156:737-43.

15 DiGiovine B, Lynch III JP, Martinez FJ, et al. Bronchoalveolar lavage neutrophilia is associated with obliterative bronchiolitis after lung transplantation. F Immunol 1996; 157:4194-202.

16 Ward C, Snell GI, Zheng L, et al. A cross sectional comparison of endobronchial biopsy and bronchoalveolar lavage: clinically stable lung allograft recipients versus recipients with bronchiolitis obliterans syndrome. Am $\mathcal{F}$ Respir Crit Care Med 1998;158:84-91.

17 Riise GC, Williams A, Kjellstrom C, et al. Bronchiolitis obliterans syndrome in lung transplant recipients is associobliterans syndrome in lung transplant recipients is associaxidant status in the lung. Eur Respir $\mathcal{F}$ 1998;12:82-8.

18 Strieter RM, Standiford TJ, Rolfe MW, et al. Interleukin-8. In: Kelley J, ed. Cytokines of the lung. New York: Marcel Dekker, 1993: 281-305

9 Ziegenhagen MW, Zabel P, Zissel G, et al. Serum level of interleukin 8 is elevated in idiopathic pulmonary fibrosis and indicates disease activity. Am $\mathcal{F}$ Respir Crit Care Med 1998;157:762-8.

20 Henke JA, Golden JA, Yelin EH, et al. Persistent increases in BAL neutrophilia as a predictor of mortality following lung transplantation. Chest 1999;115:403-9.

21 Obayashi Y, Yamadori I, Fujita J, et al. The role of neutrophils in the pathogenesis of idiopathic pulmonary fibrosis. Chest 1997;112:1338-43.

22 Cooper JD, Billingham M, Egan T, et al. A working formulation for the standardization of nomenclature and for clinical staging of chronic dysfunction in lung allografts. $\mathcal{f}$ Heart Lung Transplant 1993;12:713-6.

23 Snell GI, Ward C, Wilson JW, et al. Immunopathological changes in the airways of stable lung transplant recipients. Thorax 1997;52:322-8.

24 Lacoste J, Bousquet J, Chanez P, et al. Eosinophilic and neutrophilic inflammation in asthma, chronic bronchitis, and chronic obstructive pulmonary disease. $\mathcal{F}$ Allergy Clin Immunol 1993;92:537-48.

25 Mio T, Romberger DJ, Thompson AB, et al. Cigarette smoke induces interleukin-8 release from human bronchial epithelial cells. Am f Respir Crit Care Med 1997;155:1770-6.

26 Koch AE, Polverini PJ, Kunkel SL, et al. Interleukin 8 (IL-8) as a macrophage-derived mediator of angiogenesis. Science 1992;258:1798-801.

27 Smith DR, Polverini PJ, Kunkel SL, et al. F Exp Med 1994; 179:1409-15.

28 Keane MP, Arenberg DA, Lynch JP, et al. The CXC chemokines, IL-8 and IP-10, regulate angiogenic activity in idiopathic pulmonary fibrosis. I Immunol 1997;159:1437-43.

29 Zheng L, Orsida B, Ward C, et al. Airway vascular changes in lung allograft recipients. I Heart Lung Transplant 1999;18:231-8.

30 Yousem SA, Berry GJ, Cagle PT, et al. Revision of the 1990 working formulation for the classification of pulmonary allograft rejection. Heart Lung Transplant 1996;15:1-15. 